\title{
Year in review 2013: basic science and epidemiology
}

\author{
Paul Cullinan, ${ }^{1}$ Clare M Lloyd ${ }^{2}$
}

In the second of our reviews of 2013 we reveal our prize winners in the fields of basic science and epidemiology. As before, manuscripts arising from Imperial, Leicester and Oxford are disallowed, not because we would like to level the playing field (nice as this would be) but because of editors' conflict of interest. If you read to the end we reveal our overall winners in the traditional gold, silver and bronze positions.

\section{BASIC SCIENCE}

The Basic Science for the Chest Physician section has proved popular with our readers according to our most recent survey. Although many of these pieces are commissioned to accompany particular articles, we also welcome uncommissioned articles and suggestions for potential subjects from readers. So, if there is an area of basic science that you would like to highlight for the respiratory community, please let us know.

Manuscripts within the basic science sphere incorporated those seeking to understand the molecular mechanism underlying lung diseases as well as those aimed at discovering novel biomarkers and preclinical testing of novel therapeutic pathways. We continue to publish models and systems designed to reflect the broad range of lung diseases that are of interest to the readers of Thorax, including chronic obstructive pulmonary disease (COPD), asthma, idiopathic pulmonary fibrosis (IPF) and cystic fibrosis as well as respiratory infections.

Pulmonary inflammation may occur either as a result of excessive activation of inflammatory pathways or a failure in immune regulation. Several studies this year indicated that inflammation during COPD may occur as a general failure of pulmonary immune regulation, leading to reduced regulatory $\mathrm{T}$ cell populations but increased proportions of effector Th cells such as those defined by interleukin 17

\footnotetext{
${ }^{1}$ Department of Occupational and Environmental Medicine, National Heart and Lung Institute, London, UK; ${ }^{2}$ Leukocyte Biology, NHLI, Imperial College, London, UK Correspondence to Dr Paul Cullinan, Department of Occupational and Environmental Medicine, National Heart and Lung Institute, Manresa Road, London SW3 6LR, UK;

p.cullinan@imperial.ac.uk
}

(IL-17) secretion. ${ }^{1} 2$ Similarly, aberrant epidermal growth factor receptor activity was shown to be behind the decreased expression of FoxO3A-a negative regulator of $\mathrm{NF \kappa B}$, culminating in increased IL-8 generation in mouse and human models of COPD. ${ }^{3}$

Proteases are able to modulate inflammatory pathways by cleaving proteins, thereby adjusting their inflammatory potential. Proteolytic cleavage of the naturally occurring protein elafin by the $20 \mathrm{~S}$ proteasome may account for the observed decrease in elafin observed over the course of acute lung injury and predispose people to excessive inflammatory responses. ${ }^{4}$ Similarly, circulating neutrophils from patients with COPD showed reduced chemotactic activity and capacity for bacterial killing due to the presence of elastin peptides, released as a result of proteases released from lung-infiltrating neutrophils. ${ }^{5}$ Modulation of inflammation by inhaled cowshed dust may also be due to proteolytic activity, since dust extract was found to promote release of the complement fragment C5a in murine bronchoalveolar lavage fluid, thus explaining the protective properties seen in allergic asthma. ${ }^{6}$ This very neat mechanistic study was the winner of our bronze medal. Host responses also contribute to the regulation of infection. Airway epithelial cells maintain protective antibody and B cell responses in the lung during respiratory syncytial virus (RSV) infection. ${ }^{7}$

The function of IL-17 during pulmonary immune responses is controversial, with both anti- and pro-inflammatory functions reported. In Thorax this year we contributed to this ongoing debate, reporting on the role of Th17 cells in mediating local and systemic inflammation during community-acquired pneumonia. ${ }^{8}$ In contrast, IL-17A reduces airway hyperresponsiveness induced by RSV during allergic inflammation in a mouse model. ${ }^{9}$ Resolution of this issue is particularly topical, given the lack of success in recent trials of anti-IL-17 in asthma. ${ }^{10}$

Lung remodelling is a recognised consequence of a number of lung diseases, but the underlying mechanism remains unclear. This transformation of lung structure is not restricted to just the airway epithelium. Imaging of biopsies from patients with COPD, coupled with computerised three-dimensional reconstruction, showed dendritic cell-rich alveolar-lymphoid interfaces which may provide a structural basis for an increased capacity to respond to alveolar antigens and thus contribute to aggravated inflammation seen in severe COPD. ${ }^{11}$ This terrific study was the winner of our gold medal in this category. Vascular remodelling has been shown in asthma and COPD, and was associated with increased expression of bradykinin receptors $^{12}$ while tetrahydrobiopterin (BH4) — a cofactor of nitric oxide synthasewas identified as a potential pathway promoting vascular remodelling in IPF. ${ }^{13}$ The cytokines activin A and IL-25 were identified as potential mediators of allergeninduced airway remodelling using mouse models. ${ }^{14} 15$ Cigarette smoke was found to be a major factor in influencing epithelial to mesenchymal transition of epithelial cells from patients with COPD,${ }^{16}$ with abnormalities in Wnt signalling proposed as a potential mechanism. ${ }^{17}$ Cigarette smoke was also shown to be a causative factor in adhesion of mononuclear cells to the pulmonary arterial endothelium via fractalkine and its receptor CX3CR1, indicating that targeting this axis might reduce COPD-associated cardiovascular disorders. $^{18}$

Many diseases are heterogeneous in nature and it is hoped that more sophisticated identification of clinical subtypes may improve diagnosis and treatment. Molecular characterisation of clinical samples has revealed new subtypes of IPF, defined by expression of cilium-associated genes, ${ }^{19}$ while identification of BCG-specific IgG secreting peripheral plasmablasts as a potential biomarker may improve diagnosis of active tuberculosis (TB), particularly in those patients who also have HIV. $^{20}$

Although in Thorax we publish a number of preclinical studies using in vivo disease models, we ask authors to stress the clinical relevance of the work and to highlight the applicability of their results to treatment of lung disease in patients. Delivery of human umbilical cord-derived stem cells prophylactically or therapeutically into airways of newborn rats exposed to hypoxia showed short-term effects in partially preventing and rescuing lung function and structure. Moreover, cellfree conditioned supernatant also demonstrated therapeutic benefit and showed long-term improvement in exercise capacity and lung structure in experimental bronchopulmonary dysplasia. ${ }^{21}$ Conversely, a preclinical study of a selective small molecule inhibitor in a primate 
model of asthma showed that antagonism of the chemokine receptor CCR8 is ineffective. ${ }^{22}$ This very nicely done but comprehensively negative study-the winner of our silver medal-was accompanied by a thought-provoking editorial by Roberto Solari who discussed the value of publishing negative results in general and for the drug discovery process in particular, as well as adding to the debate regarding suitable animal models of asthma. ${ }^{23}$

\section{EPIDEMIOLOGY}

Some would say that, without tobacco, TB and inhalers, most respiratory physicians would not need to get out of bed in the morning. The epidemiology we published in 2013 did not do much to dispel this canard.

In July we published some findings of the 26-year follow-up of the Tucson birth cohort-or, at least, findings for the $40 \%$ of participants still living in the city or willing to travel to Arizona for measurement of their lung function. ${ }^{24}$ Those who had at any point admitted to smoking and those whose parents were smokers had spirometry that was no different from that of those who found themselves in neither of these camps. In contrast, those with a 'double hit'-that is, children of smokers and also smokers themselves-had evidence of impending airflow obstruction with a forced expiratory volume in $1 \mathrm{~s} /$ forced vital capacity ratio reduced by about 3\% and a faster decline over the preceding 15 years. The authors suggest that exposure to smoking at home in early life primes the lung to be hypersensitive to tobacco smoke; an alternative explanation is that the apparent synergy reflects one or more unmeasured socioeconomic determinants of adult lung function for which parental smoking is simply a marker.

The British have fewer qualms about measuring 'socioeconomic status'; indeed, some would say it is as natural to them as losing at sports they invented. The Nottingham group used it, alongside a clutch of other demographic variables and patterns of consultations, in a statistical model that was able to 'predict' lung cancer rather better, the authors claim, than that recommended in the UK referral guidelines. $^{25}$ We need to learn how to detect lung cancer earlier, an issue also raised by the London authors of a stagebased comparison of survival in six countries including the UK, ${ }^{26}$ but it is not easy to see how. At one cut-off the Nottingham model displays an impressive sensitivity of about 95\%, but this is accompanied by a false-positive rate of about 50\% which would make for a huge number of needlessly investigated and frightened patients.

There was a more cheerful message from the Tobacco Control Research Group in Bath, UK with news that the passage of smoke-free legislation in England in 2007 seems to have been followed by a $5 \%$ annual reduction-equivalent to about 2000 events a year-in adult hospital admissions to hospital for asthma. $^{27}$ Remarkably, the effect was immediate. For those, post-legislation, who are tired of smoking on street corners, new help may be soon at hand. In November we published a small systematic review of the efficacy and safety of cytisine as an aid to smoking cessation. $^{28}$ Cytisine is produced from the leaves of Golden Rain (laburnum to you and me), which the people of the Balkans used as a tobacco substitute during the privations of World War II and which, for almost 50 years, has been manufactured and sold by a Bulgarian pharmaceutical company under the catchy trade name Tabex. The stuff seems both effective and safe and is much cheaper than most alternatives but, because its evidence base would probably fail most current legislative hurdles this side of Europe (and the USA) and because nobody stands to make much money out of it, it may struggle to get into the wider pharmacopoeia. For a thought-provoking and beautifully written manuscript, we award this paper our gold medal in this section.

The wider use of interferon-gamma release assays in the identification of (latent) TB was nicely demonstrated in two manuscripts we published: first, as an effective substitute for chest radiography in screening immigrants from countries with high rates of $\mathrm{TB}^{29}$ in which case it is most cost-effective where a screening threshold is set at an 'intermediate' prevalence; and second, ${ }^{30}$ as a simple one-step screen for adult TB contacts in whom the 'number needed to screen' to detect a single case is about 35. These were neat pragmatic studies with a real potential to change practice; more of the same please. From elsewhere we learned more sobering news. Almost one-third of newly diagnosed patients with TB in southern Mexico had diabetes; those with both conditions had more extensive disease that was slower to respond to treatment and more likely to recur or relapse. ${ }^{31}$ With a rapidly increasing incidence of diabetes in most parts of the world and our continuing failure to contain the Captain of Death, this is a problem that ought to be near the top of many public health agendas. This didn't do much to cheer us but we're giving it the 2013 silver medal anyway.

And so to inhalers. In 2013 we learnt again that women who use inhaled longacting $\beta$ agonists or steroids, at low or moderate doses, to treat their asthma while they are pregnant do not need to fear that this will harm their baby, ${ }^{32}$ but there is some residual concern over higher doses of steroids. From Canada we were reminded that the use of inhaled steroids in patients with COPD increases their risk of developing severe pneumonia, $^{33}$ an effect that may be peculiar to fluticasone. An opinion piece from the same centre suggested that the number of patients so treated 'needed' to induce one case of pneumonia may be as low as $15-20 .^{34}$ If these findings are anywhere near the truth, then we will need to do some serious re-thinking about the value of these drugs in a disease whose worldwide prevalence is set only to increase.

We enjoy publishing high-quality papers on occupational disease (and would like to see more submitted) because interventions in the workplace offer real chances to effect immediate improvements to respiratory health. A neat study of Indian goldsmiths confirmed the pulmonary toxicity of cadmium fume, an established cause of emphysema ${ }^{35}$; we recognise the incongruity but are delighted to award the authors our bronze medal. More tenuously, perhaps, an analysis of the British 1958 birth cohort ${ }^{36}$ reported 18 occupations with an enhanced risk of asthma, many of them previously identified. Seven included the likely use of 'cleaning' products, leaving us still with the question of whether epidemiologically-identified respiratory symptoms in cleaners are what most of us would recognise as 'asthma'.

Finally, the year provided us with three resolutions that our chief editors might like to adopt for 2014: sort out your telomeres which aren't getting any longer ${ }^{37}$; cut back on the Valium because it will give you a nasty chest $^{38}$; and cut out the cod liver oil altogether since it will only give you asthma. ${ }^{39}$

\section{Competing interests None}

Provenance and peer review Not commissioned; internally peer reviewed.

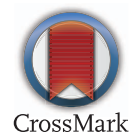

To cite Cullinan P, Lloyd CM. Thorax 2014;69:505507.

Accepted 12 March 2014

Published Online First 28 March 2014 
Thorax 2014:69:505-507.

doi:10.1136/thoraxjnl-2014-205423

\section{REFERENCES}

1 Hou J, Sun Y, Hao Y, et al. Imbalance between subpopulations of regulatory T cells in COPD. Thorax 2013;68:1131-9.

2 Maneechotesuwan K, Kasetsinsombat K, Wongkajornsilp $A$, et al. Decreased indoleamine 2,3-dioxygenase activity and IL-10/IL-17A ratio in patients with COPD. Thorax 2013;68:330-7.

3 Ganesan S, Unger BL, Comstock AT, et al. Aberrantly activated EGFR contributes to enhanced IL-8 expression in COPD airways epithelial cells via regulation of nuclear FoxO3A. Thorax 2013;68:131-41.

4 Kerrin A, Weldon S, Chung AH-K, et al. Proteolytic cleavage of elafin by $20 \mathrm{~S}$ proteasome may contribute to inflammation in acute lung injury. Thorax 2013;68:315-21

5 Dupont A, Dury S, Gafa V, et al. Impairment of neutrophil reactivity to elastin peptides in COPD. Thorax 2013:68:421-8.

6 Stiehm M, Bufe A, Peters M. Proteolytic activity in cowshed dust extracts induces $\mathrm{C} 5$ a release in murine bronchoalveolar lavage fluids which may account for its protective properties in allergic airway inflammation. Thorax 2013:68:31-8.

7 McNamara PS, Fonceca AM, Howarth D, et al. Respiratory syncytial virus infection of airway epithelial cells, in vivo and in vitro, supports pulmonary antibody responses by inducing expression of the B cell differentiation factor BAFF. Thorax 2013:68:76-81.

8 Paats MS, Bergen IM, Hanselaar WEJJ, et al. T helper 17 cells are involved in the local and systemic inflammatory response in community-acquired pneumonia. Thorax 2013:68:468-74.

9 Newcomb DC, Boswell MG, Reiss S, et al. IL-17A inhibits airway reactivity induced by respiratory syncytial virus infection during allergic airway inflammation. Thorax 2013;68:717-23.

10 Busse WW, Holgate $\mathrm{S}$, Kerwin E, et al. Randomized, double-blind, placebo-controlled study of brodalumab, a human anti-IL-17 receptor monoclonal antibody, in moderate to severe asthma. Am J Respir Crit Care Med 2013:188:1294-302.

11 Mori M, Andersson CK, Svedberg KA, et al. Appearance of remodelled and dendritic cell-rich alveolar-lymphoid interfaces provides a structural basis for increased alveolar antigen uptake in chronic obstructive pulmonary disease. Thorax 2013:68:521-31
12 Ricciardolo FLM, Sabatini F, Sorbello V, et al. Expression of vascular remodelling markers in relation to bradykinin receptors in asthma and COPD. Thorax 2013;68:803-11.

13 Almudéver $\mathrm{P}$, Milara J, De Diego A, et al. Role of tetrahydrobiopterin in pulmonary vascular remodelling associated with pulmonary fibrosis. Thorax 2013;68:938-48.

14 Hardy CL, Nguyen H-A, Mohamud R, et al. The activin $A$ antagonist follistatin inhibits asthmatic airway remodelling. Thorax 2013;68:9-18

15 Gregory LG, Jones CP, Walker SA, et al. IL-25 drives remodelling in allergic airways disease induced by house dust mite. Thorax 2013:68:82-90.

16 Milara J, Peiró T, Serrano A, et al. Epithelial to mesenchymal transition is increased in patients with COPD and induced by cigarette smoke. Thorax 2013;68:410-20.

17 Heijink $I H$, de Bruin $H G$, van den Berge $M$, et al. Role of aberrant WNT signalling in the airway epithelial response to cigarette smoke in chronic obstructive pulmonary disease. Thorax 2013:68:709-16.

18 Rius C, Company C, Piqueras L, et al. Critical role of fractalkine (CX3CL1) in cigarette smoke-induced mononuclear cell adhesion to the arterial endothelium. Thorax 2013;68:177-86.

19 Yang IV, Coldren CD, Leach SM, et al. Expression of cilium-associated genes defines novel molecular subtypes of idiopathic pulmonary fibrosis. Thorax 2013;68:1114-21.

20 Ashenafi S, Aderaye G, Zewdie M, et al. BCG-specific lgG-secreting peripheral plasmablasts as a potential biomarker of active tuberculosis in HIV negative and HIV positive patients. Thorax 2013;68:269-76.

21 Pierro M, Ionescu L, Montemurro T, et al. Short-term, long-term and paracrine effect of human umbilical cord-derived stem cells in lung injury prevention and repair in experimental bronchopulmonary dysplasia. Thorax 2013;68:475-84.

22 Wang $\mathrm{L}$, Jenkins TJ, Dai $\mathrm{M}$, et al. Antagonism of chemokine receptor CCR8 is ineffective in a primate model of asthma. Thorax 2013:68:506-12.

23 Solari R. Another chemokine target bites the dust? Thorax 2013:68:501-3.

24 Guerra S, Stern DA, Zhou M, et al. Combined effects of parental and active smoking on early lung function deficits: a prospective study from birth to age 26 years. Thorax 2013;68:1021-8.

25 Iyen-Omofoman B, Tata LJ, Baldwin DR, et al. Using socio-demographic and early clinical features in general practice to identify people with lung cancer earlier. Thorax 2013;68:451-9.
26 Walters S, Maringe C, Coleman MP, et al. Lung cancer survival and stage at diagnosis in Australia, Canada, Denmark, Norway, Sweden and the UK: a population-based study, 2004-2007. Thorax 2013:68:551-64.

27 Sims M, Maxwell R, Gilmore A. Short-term impact of the smokefree legislation in England on emergency hospital admissions for asthma among adults: a population-based study. Thorax 2013;68:619-24.

28 Hajek P, McRobbie $H$, Myers K. Efficacy of cytisine in helping smokers quit: systematic review and meta-analysis. Thorax 2013;68:1037-42.

29 Pareek M, Bond M, Shorey J, et al. Community-based evaluation of immigrant tuberculosis screening using interferon $\gamma$ release assays and tuberculin skin testing: observational study and economic analysis. Thorax 2013;68:230-9.

30 Haldar $\mathrm{P}$, Thuraisingam $\mathrm{H}$, Patel $\mathrm{H}$, et al. Single-step QuantiFERON screening of adult contacts: a prospective cohort study of tuberculosis risk. Thorax 2013;68:240-6.

31 Jiménez-Corona ME, Cruz-Hervert LP, García-García L, et al. Association of diabetes and tuberculosis: impact on treatment and post-treatment outcomes. Thorax 2013:68:214-20.

32 Cossette B, Forget A, Beauchesne M-F, et al. Impact of maternal use of asthma-controller therapy on perinatal outcomes. Thorax 2013;68:724-30.

33 Suissa S, Patenaude V, Lapi F, et al. Inhaled corticosteroids in COPD and the risk of serious pneumonia. Thorax 2013;68:1029-36.

34 Suissa S. Number needed to treat in COPD: exacerbations versus pneumonias. Thorax 2013:68:540-3.

35 Moitra S, Blanc PD, Sahu S. Adverse respiratory effects associated with cadmium exposure in small-scale jewellery workshops in India. Thorax 2013:68:565-70.

36 Ghosh RE, Cullinan P, Fishwick D, et al. Asthma and occupation in the 1958 birth cohort. Thorax 2013:68:365-71.

37 Rode L, Bojesen SE, Weischer M, et al. Short telomere length, lung function and chronic obstructive pulmonary disease in 46396 individuals. Thorax 2013:68:429-35.

38 Obiora E, Hubbard R, Sanders RD, et al. The impact of benzodiazepines on occurrence of pneumonia and mortality from pneumonia: a nested case-control and survival analysis in a population-based cohort. Thorax 2013:68:163-70.

39 Mai X-M, Langhammer A, Chen Y, et al. Cod liver oil intake and incidence of asthma in Norwegian adults —the HUNT study. Thorax 2013;68:25-30. 